CAHIERS DE

NARRATOLOGIE

\section{Cahiers de Narratologie}

Analyse et théorie narratives

36 | 2019

Rhétorique et représentations de la culture mafieuse.

Images, rituels, mythes et symboles

\title{
Au long de l'histoire du mystère : la mafia s'épanchant en mythe
}

\section{Laurent Lombard}

\section{(2) OpenEdition \\ Journals}

Electronic version

URL: http://journals.openedition.org/narratologie/9611

ISSN: $1765-307 X$

\section{Publisher}

LIRCES

\section{Electronic reference}

Laurent Lombard, « Au long de l'histoire du mystère : la mafia s'épanchant en mythe », Cahiers de Narratologie [Online], 36 | 2019, Online since 20 December 2019, connection on 20 December 2019. URL : http://journals.openedition.org/narratologie/9611

This text was automatically generated on 20 December 2019.

Article L.111-1 du Code de la propriété intellectuelle. 


\title{
Au long de l'histoire du mystère : la mafia s'épanchant en mythe
}

\author{
Laurent Lombard
}

1 Rarement, du début du XXe siècle à nos jours, un phénomène aura été aussi présent dans le débat et l'imaginaire collectifs (nationaux, européens, mondiaux soient-il), tout en étant mal connu, que celui des mafias. A telle enseigne que, au-delà de la scabreuse et sinistre actualité, donc de la réalité, qui nous parvient parfois, entre ce que l'on sait et ce que l'on ne sait pas, entre ce qui se dit et ce qui s'invente, s'est formée une véritable mythologie de la mafia; il ne serait d'ailleurs pas abusif d'alléguer que la mafia est devenue une sorte de mythe moderne ${ }^{1}$. En ce sens qu'on se fait d'elle une représentation souvent fausse, parfois idéalisée, qui alimente l'imagination. En ce sens encore où elle suscite des légendes : l'évocation de certains faits, idées, ou personnes ayant trait à la mafia peut être transformée en légende de la force, du pouvoir, et du coup, donnant confiance, incite à une identification et, pour certains, à en rejoindre les rangs. En ce sens enfin où la mafia n'est ni une idéologie ni un concept, mais plutôt un système d'actions et de communications, un message, avec ses propres signes, signifiants et signifiés, ce qui correspond quelque peu à la définition que Roland Barthes a donné du mythe ${ }^{2}$.

2 Quel est le rapport exact que l'on peut établir entre mafia, mythe et communication? Si le mythe est parole, toujours selon Barthes, et si la mafia est silence, selon l'image qu'on s'en fait, on comprend dès lors que ce rapport va s'établir entre parole et silence, outre à ce qui unit la parole et le silence, en l'occurrence ici, le secret, qui instaure le sacré (ce n'est pas un hasard si les deux termes possèdent une racine commune). Autrement dit, ce qui est central dans le rapport mafia-mythe et communication, c'est le mystère, dont la racine grecque $\mu v ́ \omega$ (muô) «se taire » est commune avec «muet ", mais également «mythe ». Du mystère, la mafia en a même fait son emblème et son étendard, à savoir son moyen de communication. C'est bien là effectivement le point nodal qui conduit les mafias à s'épancher stratégiquement en mythe, suivant les traces de certaines traditions longues. Il faut dès lors accepter que les organisations criminelles mafieuses se placent sous la figure d'Harpocrate, d'Horus, fils d'Isis et 
d'Osiris, appelant à la discrétion initiatique les adeptes des cultes à mystères, si l'on en croit du moins les poètes classiques, dont Catulle par exemple, qui n'hésitent pas pour certains à le rapprocher d'Apollon et de Dionysos, le dieu des Mystères précisément, qui a par ailleurs aussi affinité avec les puissances démoniaques (ce qui est, on peut le dire, une caractéristique de la mafia). Il faut accepter que les mafias entretiennent un lien avec les traditions de l'Antiquité qui savaient que, par définition, il n'est point de mystes, d'initiés, sans aptitude au secret et au silence. C'est en grande partie sur cette propriété fondamentale que les mafias basent leur système de communication. Le mystère (englobant secret et silence) qui paraît à première vue équivaloir à une absence de communication, est en effet investi d'une dimension communicationnelle intra et extra communautaire. Intra, car le mystère a une fonction cohésive au sein du groupe et devient fédérateur en engendrant un sentiment de connivence, de complicité et de confiance mutuelle entre les membres qui le conservent ${ }^{3}$. Extra, car la pratique du mystère s'inscrit dans une stratégie de communication avec l'extérieur afin de happer de futurs initiés. En ce cas, il fonctionne comme technique de pouvoir qui produit des effets de discrimination mais aussi, notamment chez les plus jeunes, comme nous le verrons, d'identification. Ainsi l'aura de mystère qui entoure les organisations mafieuses peut leur offrir une puissance performative stratégique, laquelle ne sera pas étrangère à la mythisation de telles sociétés secrètes. Au fond, le coup de génie en matière de communication de ces organisations, c'est que, en dépit de nos sociétés qui ont évolué vers un tout-communicationnel, vers l'avènement et la tyrannie du bruit ${ }^{4}$ et de la transparence ${ }^{5}$ à tout crin, elles restent proches de certaines grandes traditions, qui en demeurant dans l'ombre du mystère et du secret, rendaient banal ce qui était discret pour ne pas être dévoilé, tout en créant une fascination et/ou une répulsion naturelle: réactions normales dues à la méconnaissance de ce que les mafias sont véritablement.

3 Cette communication de l'incommunicable fabrique de l'interprétation - tout mythe n'est-il pas origine et effet d'une interprétation? -, donc fait parler. D'autant plus lorsque le langage de ces organisations est un langage «trouble et prudent, presque codé $»^{6}$ qui nécessite doublement une herméneutique ${ }^{7}$. Le mythe est parole (il fait parler), disait justement Barthes, la mafia l'est aussi. Le mythe est message, la mafia également. Et ainsi que le précisait le juge Giovanni Falcone: «l'interprétation des signes, des messages constitue l'une des principales activités des hommes d'honneur $»^{8}$.

Cette expression d'«hommes d'honneur", ainsi qu'on appelle et que s'appellent généralement les affiliés des organisations mafieuses, pourrait paraitre paradoxale puisqu'elle définit des hommes vertueux, intègres qui ne transigent nullement avec les lois les plus strictes de la morale. Mais il s'agit d'un parfait exemple de message communicationnel visant à enjoliver et à brouiller, pour la vitrine, l'image de la réalité violente et criminelle de ces organisations". Cette expression "homme d'honneur ", si simple et qui est devenue une évidence presque à la mode, permet ainsi aux mafias de s'inscrire avantageusement dans la suite des grandes traditions et légendes où courage, exploits, bravoure et honneur étaient les maîtres mots, et de se parer d'une image mythico-héroïque; celle-là même qui deviendra pour certains objet de désir. Car la communication mafieuse, comme nous le verrons, joue savamment entre frustration sociale et désir d'identification héroïque. Quête éternelle de l'argent et du pouvoir. Comme deux piliers massifs ayant pour chapiteaux des scènes légendaires, et qui soutiennent des arcs obscurs qui enjambent d'incroyables vides, où va se justifier le choix de la criminalité comme mode de vie honorable. Paradoxe s'il en est, certes, mais 
la communication des mafias se construit sur une représentation inadéquate qui remplit cependant une fonction nécessaire. Ainsi, par exemple, le mot « honneur » est passablement détourné de son sens mais prend une valeur centrale, à la mesure de ce qu'en écrivait le romancier Octave Feuillet : «L'honneur, dans son caractère indéfini, est quelque chose de supérieur à la loi et à la morale : on ne le raisonne pas, on le sent. C'est une religion $»^{10}$. Aussi, derrière l'expression "homme d'honneur», il faut également entendre une invitation à l'obéissance, à la fidélité ${ }^{11}$ afin de créer un lien (religion), une alliance, tout autant qu'à tenir sa parole. Tenir sa parole, n'est-ce pas avant tout garder le silence? L'honneur est à tel point important que forfaire à la valeur de l'honneur implique bien souvent une vengeance létale ${ }^{12}$ qui est elle-même d'ailleurs communication ${ }^{13}$.

5 Outre cette communication ingénieuse intra et extra communautaire, ce qui semble fonder la mythologie mafieuse ou tout du moins donner aux sociétés secrètes dites mafieuses un halo mythologique, c'est bien l'opacité, le mystère de leur fonctionnement et, partant, le silence de leurs affiliés. La loi du silence s'impose et s'expose. Mais au fond, qu'est-ce à dire cette loi du silence ? N'est-elle pas elle aussi une (ré)appropriation communicationnelle? Cette expression est devenue elle aussi d'un usage fort répandu pour indiquer généralement ce qui doit être tenu secret par une ou plusieurs personnes. Mais quelle est son histoire, entendons : qui l'a instaurée pour la première fois ? Est-elle une création de la modernité ? Que signifie-t-elle? Pour le dictionnaire, c'est "l'interdiction de donner, notamment à la police, des renseignements confidentiels $»^{14}$. Définition pour le moins vaste sinon vague. Il serait alors plus juste, sans doute, pour ce qui concerne les mafias, de parler de " conspiration du silence " dont la définition est: "pacte d'honneur par lequel un ensemble de personnes s'engage à ne pas divulguer ce qui doit être tenu secret $\aleph^{15}$. Or s'est imposée l'expression « loi du silence ». Cela n'a-t-il pas profité aux mafias d'ailleurs, et/ou n'en sont-elles pas à l'origine, car le mot «loi » demeure dans la sphère de la connotation positive voire du sacré16, à l'inverse du terme "conspiration». Quelle valeur donc accorder à cette expression? Car peut-on empêcher la parole de circuler? Peut-on vraiment dire l'ineffable ? Cela étant, l'Homme est-il capable de silence, c'est-à-dire de ne pas parler, de ne pas faire de bruit, pour quelque cause que ce soit? L'humain sait retenir le $\mathrm{Verbe}^{17}$, les informations, mais ne ressent-il pas le besoin de les divulguer à un moment ou à un autre? Est-ce que le dire n'est pas la source d'un quelconque pouvoir? Je sais, donc j'ai un avantage sur autrui. Celui de dire, ou de ne pas dire. Surtout, dans nos sociétés où l'essor des systèmes technologiques et informatiques, de communication, de réseaux sociaux a apparemment considérablement ouvert à ce qui pouvait être secret ou à ce qui doit/devait être « tenu secret ", la loi du silence s'impose-t-elle et s'expose-t-elle encore ou bien n'est-elle devenue qu'un symbole, relégué à un simple rôle d'élément de rites anciens ? ${ }^{18}$

En définitive, ce qui doit nous intéresser dans cette expression, c'est la façon dont elle est devenue en quelque sorte synonymique du terme mafia. C'est que la mafia et la loi du silence ont été en tout temps et en tout lieu placées sous l'idée d'un rite. Sémiologiquement la mafia est un système de signes, quelque iconiques ou symboliques qu'ils soient. Nombre de ces signes sont peu ou prou ceux de la loi du silence.

7 Plus précisément, la mafia est sémiologiquement parlant un système de signes mixte : elle comporte des signes linguistiques oraux et des signe symboliques et/ou iconiques (type d'actions, mimique de reconnaissance, gestes et façons de tuer laissant apparaître 
une éventuelle symbolique de vengeance...). Le silence peut être lui aussi, sémiologiquement parlant, un système de signes iconiques et/ou symboliques où les gestes et les mimiques, isolés de phonèmes, de la parole, prennent toute leur valeur. Cela impose au préalable qu'une parole, qu'un discours aient été dits, qui doivent être tenus secrets. Donc on peut en déduire que pour le silence aussi, il s'agit d'un système de signes mixte (oraux et iconiques).

8 La sémiologie de la mafia comme celle de la loi du silence impliquent donc des codes, un comportement, qui ne sont pas privés de la conscience de leur propre ritualité. Une sémiologie qui est au principe de tous les rituels à mystères et qui fait qu'on pourrait même parler d'une « ritualité naturelle ».

9 La force des rites à mystères est qu'ils ont su évoluer, s'adapter à toutes les situations historiques. Il en va ainsi par exemple des rites religieux, chrétiens en l'occurrence ${ }^{19}$, ou des rites maçonniques ${ }^{20}$, pour ne citer que des familles de rites avec lesquels parfois on a cru observer des convergences avec les rites mafieux, par la place qu'y tenait le silence, par la connivence qu'on pouvait déceler entre le sacré et le secret.

10 Si étrange que cela puisse paraître, un mot simple semble relier ces rites : l'humilité. Si l'on part en effet de l'une des étymologies possibles du mot «omertà », par lequel on a fini par désigner en Italie la loi du silence liée aux associations criminelles, et qui viendrait de omo " homme » et umirtà, contraction de umiltà " humilité " ${ }^{21}$, on devine alors une allusion au Christ, le plus humble de tous; cette même allusion qui est reprise dans une citation du rituel maçonnique au $18^{\mathrm{e}}$ degré du Rites Ecossais Ancien et Accepté2 $^{2}$. Cette "humilitas", à l'enseigne du mot "honneur", comme on l'a vu précédemment, implique historiquement et rituellement à la fois une discrétion, une obéissance et une fidélité, une foi en somme ${ }^{23}$ vis-à-vis d'une personne, d'une idée, d'une valeur, d'une organisation... On la retrouve, par exemple, tant dans les rites chevaleresques (par lesquels les mafias veulent parfois se valoriser), que dans les rites maçonniques. Si elle est clairement exprimée dans certains Hauts Grades, notamment au $18^{\mathrm{e}}$ degré ainsi que nous l'avons cité précédemment, elle est, d'une façon plus générale, au cœur des rituels des francs-maçons avec la symbolique majeure que représente la quête de la Parole perdue et, après le meurtre d'Hiram, la mise en scène de l'exécution des mauvais compagnons qui, par manque d'humilité, et donc d'allégeance au respect du secret et des règles, ont tué le Maître ${ }^{24}$.

11 Toutefois, si le terme « humilitas » rapproche implicitement ces rites, le sens des rituels mafieux se distinguent à l'évidence du rite religieux et maçonnique en ce que l'action réelle des mafias est étrangère à une quelconque idée de quête métaphysique, culturelle ou personnelle, à toute idée d'apocalypse au sens de révélation, de dévoilement. En effet, les rites à mystères tels que ceux du christianisme et de la francmaçonnerie, impliquent un mouvement ascensionnel, une montée de connaissance en connaissance, une "gnose jusqu'à une épignose", dirait Saint Paul ${ }^{25}$. Le silence, le secret, le mystère, dans ce contexte, deviennent donc Connaissance, tropologique sinon anagogique, qui doit mener aux bonnes œuvres, au discernement, à une vie morale. Connaissance comme expression d'une foi vivante en relation avec la charité fraternelle, l'agapè. Alors, bien que d'aucuns aient voulu voir un rapprochement entre ces institutions et la mafia, bien que les mafias, notamment italiennes, tentent de valoriser leur image à travers une communication directe ou indirecte sur un soi-disant ésotérisme et, comme on l'a vu, une appartenance à une tradition longue, afin de s'auto-auréoler d'une image mythique, on est loin en ce qui les concerne de tout 
mouvement spirituel ascensionnel, de toute dimension anagogique de la connaissance. Les mafias ne créent pas de théologie, tout au plus de la doxa à visée communicationnelle. Pour celles-ci, il s'agit plutôt du monde tel qu'il est vraiment, et de la façon dont il faut y prospérer. Même si tout cela est placé sous le signe du mal, du crime, de la méchanceté. On pourrait d'ailleurs certainement parler pour la mafia d'un « rite du méchant ». La sacralité n'y est plus liée à la morale, mais à l'immorale. Entre sacralité et immoralité, se déploie un aspect de la communication des mafias qui, comme nous l'avons évoqué, joue savamment entre frustration sociale et désir d'identification héroïque. La violence y est utilitaire et fonctionnelle. Cependant, les mafias justifient, légitiment leur propre violence au nom d'une cause si prosaïque qu'elle soit. Dès lors, elles s'inscrivent (volontairement ou non) dans une histoire longue puisque cet apparent paradoxe de la violence et du sacrée ${ }^{26}$ a toujours existé, quelles que soient les époques et les civilisations: les récits des mythes anciens fonctionnent entre ces deux pôles, comme nombre de textes religieux fondateurs, dont la Bible bien évidemment. L'idée du sacré, même dans la violence, est donc nécessaire aux mafias car c'est le sacré qui donne à l'homme, même criminel, son identité, sa dignité, qui le conduit à sacrifier sa propre vie (ou plutôt celle des autres!). Car, à travers le sacré et la violence, il vit cette pulsion duelle propre à chaque existence selon Freud, qu'est la pulsion de vie et la pulsion de mort. On observera, en passant, que le terrorisme politico-religieux est la forme ultime de ce phénomène.

L'attraction des contraires est selon nous essentielle pour comprendre ce que l'on pourrait appeler le "désir de mafia » des impétrants. Sans doute que les mafias, de façon plus ou moins consciente, ont compris que pulsion de vie et pulsion de mort ne sont pas antithétiques mais complémentaires, qu'elles sont prises dans un mouvement d'unification et de désunification permanente qui crée justement le désir, la pulsion. Il en va de même pour la violence et le sacré. Nous serions même tenté de dire, pour mieux comprendre, que violence et sacré se trouvent unis et désunis comme les deux principes antithétiques qui, selon le philosophe Empédocle, président à l'agencement

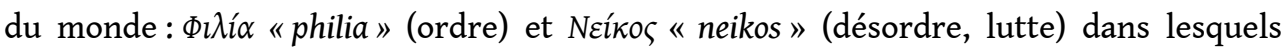
Freud avait justement trouvé une équivalence à nos pulsions. Les mafias, loin du point de vue cosmogonique d'Empédocle, entre leur volonté de valorisation par une pseudo appartenance à des traditions anciennes et le but de leur organisation, semblent toutefois fonctionner elles aussi sur cet agencement entre philia et neikos ${ }^{27}$, qui se trouve être sans nul doute le ressort de tous les récits anciens aux origines des mythes. Le mythe, au sens général du terme, pourrait donc bien se situer dans l'identification pulsionnelle entre philia et neikos. Le mythe est parole, le mythe est message mais il est aussi désir. Le mythe comme désir et le désir de mythe sont présents à toutes les époques, puisque le mythe est, bien que fondé sur une représentation apparemment faussée de la réalité d'ici bas, le reflet de nos pulsions individuelles et collectives. Nous pouvons donc penser que le désir de mafia participe de ce même schéma. C'est toute la communication et toute la sémiologie mafieuse qui répercutent ce sentiment de désir, entre violence et sacré. Les mafias criminelles modernes, nées rappelons-le à l'époque de l'avènement d'un certain capitalisme, ont certainement bien compris, avant même que le monde de la production et de la consommation de masse fasse que tout devienne objet de désir (et de frustration), qu'il fallait créer le désir. Le désir est une force agissante (tout autant qu'une force de communication) qui inspire à de nombreux jeunes marginalisés de rendre témoignage de leur foi envers une organisation criminelle. Un certain pouvoir de la mafia, qui outre à la main mise sur des territoires, 
se situe là : faire en sorte que la population sous contrôle soit emplie de désir, non pas de connaissance ni de culture, mais de désir. Les habitants (notamment les plus jeunes), souvent issus de quartiers défavorisés et pris dans une spirale de ressentiment et de colère, doivent croître dans un désir de mafia, par ce même désir d'une mafia grâce à laquelle ils espèrent réussir socialement. La mafia est vue comme un moyen pour s'en sortir, pour se démarginaliser, en tout cas d'épancher sa colère.

Comme le mythe, comme la globalisation, la mafia est une machine à désir, de ce désir qui sonne bien souvent comme un désir de puissance (et l'on retrouve ici l'héroïsme ou le désir d'identification héroïque), créé à partir de tout un imaginaire, de toute une série de représentations (à valeur communicationnelle). Le problème reste que les représentations ne correspondant pas de façon adéquate aux réalités, souvent certains " se trouvent entraînés à des jugements fautifs et, obéissant à la force des choses qu'on croit dominer, finissent par réaliser le contraire du désirable et à se retrouver en état d'impuissance ${ }^{28}$. Ce désir de mafia est donc une expression inadéquate du désir de puissance qui s'appuie sur un fantasme, un objet imaginaire ${ }^{29}$ ou pour le moins trompeur.

Il n'en demeure pas moins que par cette économie et cette politique du désir, les mafias, outre à créer des phénomènes de communication, accomplissent (qu'on le veuille ou non) un rôle social. Le désir, engendré par les représentations et les messages, par la doxa propagée par les mafias, est bel et bien utilisé comme une technique sociologique et une forme de l'action ${ }^{30}$. Le rite du méchant, tel que nous l'avons nommé plus haut, est donc un rite social - ici réside toute l'ambiguïté de la prolifération des organisations mafieuses dans nos sociétés, touchant principalement les classes les plus pauvres. Cette dernière caractéristique se retrouve d'ailleurs dans l'étymologie même du terme méchant qui vient du verbe « mécheoir » signifiant « celui qui a mauvaise chance ». Tout autant que dans celle de mafia qui viendrait du toscan où il avait pour sens «misère ». On ne peut pas dire que l'action violente, criminelle et cruelle des organisations mafieuses, soit privée d'un aspect social puisqu'elle supplée à la fois un État absent pour aider les plus démunis et opère une fascination sur les plus pauvres, sur ceux qui se considèrent des malchanceux socialement, lesquels sont attirés par une expérience sinon de réussite, de puissance, en tout cas d'existence, sociale justement.

15 En Italie, où l'absence d'un État-nation vigoureux et accompli crée un espace pour l'implantation et la diffusion des sociétés criminelles, en particulier dans certaines zones comme Scampia, banlieue de Naples, la mafia est celle qui donne des réponses immédiates, notamment à ceux qu'on appelle, en territoire ultramontain, les «baby criminali » qui sont aujourd'hui en quête d'une identité, d'une existence, d'une réussite que la société n'offre pas, ainsi qu'en témoigne Davide Cerullo dans son livre Diario di un buono a nulla ${ }^{31}$. La mafia réussit en quelque sorte à se donner une image empreinte de justice contre les lois iniques de ceux qui écrasent les plus faibles. Voilà comment le mafieux, bien qu'assassin, est devenu par nécessité, par construction et stratégie communicationnelles, un défenseur du peuple, presque un héros donc, imprégnant l'ensemble du corps social, lequel peuple baptise justement le mafieux d'homme d'honneur. Là où manquent les réponses aux exigences d'une vie réussie, le mafieux offre ce que l'ordre social devrait pouvoir offrir. De nos jours, cette figure nécessaire d'homme qui « aide » les pauvres gens a pour certains une valeur absolue, centrale. En effet, pour nombre de jeunes qui vivent dans des familles à problèmes multiples, qui 
vivent plongés dans une sous-culture de la violence, le criminel devient une figure mythique à laquelle il est possible de s'identifier. Cette identification se fait notamment grâce à cette culture proliférante, presque une religion, de l'honneur, de l'homme d'honneur. La coupe de cheveux, les tatouages, une certaine façon de s'habiller sont un véritable langage qui indique une façon d'être comme les mafieux, gagnants et forts, dont il faut avoir peur et qu'il est préférable de respecter. Il s'agit d'un langage véhiculé par une esthétique de la violence. A Naples, à Scampia, on peut ainsi voir dans les rues de jeunes enfants, mais également de jeunes adultes, cheveux rasés, tatoués, portant un $\mathrm{t}$-shirt avec une inscription, un pistolet et un nom : Pablo Escobar. Le criminel devenu légende, mythe. Toujours, à Naples, à Scampia, il y eut une période, celle de la guerre des clans en 2006, où les hommes et surtout les femmes ${ }^{32}$ se faisaient tatouer sur la partie postérieure du cou, une couronne, signe de pouvoir et de contrôle sur autrui. Esthétique de la violence qui se fait langage. Celle-là même que l'on retrouve dans le commerce florissant des films ${ }^{33}$, des séries télévisées à succès qui traitent du phénomène mafieux. En Italie, par exemple, Il capo dei capi, L'onore e il rispetto, et plus récemment Gomorra - La serie. L'esthétique cinématographique de la violence vient y rencontrer la violence réelle, ce qui devient un support supplémentaire pour une autoidentification. Il en résulte un effet inattendu: ceux que les fictions représentent comme commettant des injustices, des crimes, deviennent de véritables héros faisant pulser de désir un certain public. Aussi, derrière l'apparente dénonciation du système mafieux qu'on pourrait éventuellement déceler dans ces films ou séries télévisées, comme par ailleurs dans le florilège des romans populaires sur le thème, Gomorra et autre polars, ces fictions deviennent une composante décisive de la force mafieuse, puisqu'elles participent à rendre mythique, peut-être à leur insu, l'image du mafieux ${ }^{34}$. $\mathrm{Au}$ fond, le cas Saviano en Italie est probant en ce sens, car dans ses livres censés dénoncer la criminalité organisée, l'auteur ne déconstruit pas l'imaginaire sur lequel se fonde le désir de mafia dans une partie de la population, mais le rend vivant ${ }^{35}$. C'est là toute sa limite qui, aux yeux de certains sceptiques, révoque en doute la spectacularisation de sa tête mise à prix.

Les images des personnages criminels dans les fictions, les photos de mafieux arrêtés relayées en boucle par les médias participent d'un langage contenant quelque chose qui semble bien implanté dans l'esprit des jeunes (et pas seulement). La mafia en tire son avantage : la représenter, donne force et vigueur à ses membres; la représenter lui confère une certaine aura. Les fictions, en ne parvenant pas à détruire l'imaginaire du mythe du plus fort, de l'homme d'honneur, le renforcent, voire lui donnent la légitimité de l'œuvre d'art. N'est-ce pas le même procédé qui a lieu avec la chanson populaire? Notamment dans ce phénomène italien des "neomelodici», comme Tommy Riccio dont les textes incitent à la culture de l'honneur? Ces voix du sud, des quartiers les plus pauvres, sont en effet devenues un véritable business auquel les mafieux ont prêté main forte de différentes manières, en devenant paroliers ${ }^{36}$, attachés de presse ou patrons de labels. Outre les nombreux scandales récents ${ }^{37}$, certains chanteurs de ce genre musical ont explicitement glorifié la mafia, comme l'a souligné Jamie Mackay ${ }^{38}$. Encore une fois, la popularité d'une telle musique vient de ce qu'elle intègre tous les ingrédients nécessaires à créer un désir de mafia, puisque paroles et concepts dans ces chansons sont épongés ou intériorisés dans l'imaginaire collectif, dans l'ensemble du corps social.

Le rite social mafieux se transforme ainsi en véritable business de la douleur et du traumatisme qui vient alimenter l'imaginaire et que l'imaginaire alimente après coup. 
Sans doute que tout le langage, que tout le système sémiologique mafieux se rapporte à cette pure fonction : créer un imaginaire identificatoire. Les signes (rites, chansons, photos dans les journaux, langue proprement dite même codée) sont la matière qui servira au mythe du mafieux et à son identification populaire. C'est ainsi que la mafia qui est un désir, une réalité, s'épanchant en mythe, en mythologie, est un moment de nos sociétés, un moment de l'histoire, d'une sale histoire.

\section{BIBLIOGRAPHY}

Banet, Remi (2012), « La chanson napolitaine, à l'eau de rose et au sang de la Camorra », L'obs, 21 août 2012. En ligne. URL : https://www.nouvelobs.com/rue89/rue89-monde/20120821.RUE1855/ la-chanson-napolitaine-a-l-eau-de-rose-et-au-sang-de-la-camorra.html

Barthes, Roland (1957), « Le mythe, aujourd'hui », in Mythologies, Paris, Seuil.

Cerullo, Davide (2016), Diario di un buono a nulla, Firenze, Società Editrice Fiorentina.

Cretin, Thierry (1997), Mafias du monde : organisations criminelles transnationales : actualité et perspectives, Paris, PUF.

Dumoulié, Camille (2019), Le désir, Paris, Dunod.

Feuillet, Octave (1976 [1858]), Le roman d'un jeune homme pauvre, Paris, Tallandier.

Greco, Giovanni (2011), « I segni della mafia ». En ligne. URL http:/guide.supereva.it/ organizzazioni_criminali/interventi/2000/08/10618.shtml.

Kaiser, Wolfgang (2004), « Pratique du secret », Rives méditerranéennes, $\mathrm{n}^{\circ} 17$.

Le Breton, David (1997), Du Silence. Essai d'anthropologie, Paris, Métailié.

Mackay, Jamie (2016), «L'univers impitoyable de la Pop napolitaine », En ligne. URL : https:// www.vice.com/fr/article/yvwxxw/the-dark-and-eccentric-world-of-neapolitan-pop Saubaber, Delphine (2011), « Mafia : la terre des disparus », L'Express, 14 avril 2011. En ligne. URL https://www.lexpress.fr/actualite/societe/mafia-la-terre-des-disparus_982123.html

Simmel, Georg (1998), Secret et Sociétés secrètes, Paris, Circé.

Susini, Jean et Champeyrache, Clotilde, « Mafia» (2019), En ligne. URL : https://

www.universalis.fr/encyclopedie/mafia/ (consulté le 10 août 2019)

Zarka, Yves Charles (2006), « Ce secret qui nous tient », Cités, n²6, PUF.

\section{NOTES}

1. Cette assertion vaut certainement pour les différentes mafias qui se sont développées, chacune avec ses caractéristiques, dans l'histoire des pays. Dans notre article, quelques considérations seront d'ordre général, mais pour l'essentiel l'utilisation du terme mafia, au singulier ou au pluriel, renvoie à la situation italienne. 
2. Barthes 1957, p. 219.

3. C'est ce que note en particulier Wolfgang Kaiser à propos du secret : « [...] la garantie de garder le secret ouvre, voire crée un espace de communication à l'intérieur d'un groupe de personnes qui sont "dans le secret" ", in Kaiser 2004, p. 7.

4. Cf. Le Breton 1997.

5. Cf. Zarka 2006.

6. Selon les mots du juge Ferdinando Imposimato, in Cretin 1997, p. 127.

7. De l'herméneutique à Hermès, dieu des échanges, de la communication (et des voleurs) mais aussi de l'initiation et du secret.

8. Cretin 1997, p. 128.

9. Dans une autre mesure, il en va de même pour le rituel d'initiation pratiqué par les mafias en référence plus ou moins avérée à l'ésotérisme. Soulignons le fait que depuis quelques années maintenant certaines vidéos de ces rites sont en ligne sur Internet, cf. par exemple https:// www.youtube.com/watch? $\mathrm{v}=\mathrm{OhVZqPlhr5c}$. Cela constitue certes une violation du mystère, mais parce que ces instants (volés) sont mis en ligne, telle une fiction, cela peut créer sans aucun doute un effet de désir, comme nous le verrons. On peut dès maintenant supposer que les mafias contrôlent aussi leur image et leur communication à travers les nouveaux systèmes de médiatisation, comme Internet.

10. Feuillet 1976 [1858], p. 264.

11. Voilà comment l'honneur est dès lors placé sous le patronage de Fides, qui veille par définition à la foi jurée et à la foi promise, ainsi qu'au secret des paroles confiées dans l'ombre.

12. Cette double polarité d'honneur et de vengeance se retrouve dans le terme grec ancien $\tau \iota \mu$,', timê : à la fois honneur et respect et dans un sens négatif : peine, châtiment, vengeance. Ce mot pouvait également s'utiliser pour indiquer la marque d' "honneur» envers une autorité, et le moyen de l'honorer. N'est-ce pas le principe de tout affilié envers son supérieur dans les structures hiérarchiques mafieuses? Là encore, on note donc comment le terme honneur se réfère à un modèle plus ancien que moderne. Ajoutons que la structure hiérarchique joue aussi son rôle dans la sacralisation des sociétés secrètes, dans la mesure où le terme "hiérarchie » renvoie étymologiquement au sacré.

13. La violence et la façon de l'exercer est, pour de nombreuses mafias, un message à l'adresse des forces de l'ordre, du pouvoir et des affiliés. Ainsi, par exemple, l'utilisation de la lupara indique-t-elle la trahison du clan et l'expression lupara bianca, un crime avec disparition du cadavre (cf. Saubaber 2011) ; ou bien les signes laissés sur un cadavre : « une main coupée sur le cadavre : cela voulait dire que le mort avait volé, en sachant qu'il ne devait pas voler dans cette zone-là ou bien ne pas voler la victime protégée par la mafia; les yeux arrachés et fermés dans une main, ça signifiait que le mort avait tué quelqu'un en lien avec la mafia; une raquette de figuier de barbarie sur la poitrine que le mort s'était emparé d'argent qui ne lui revenait pas. Ou bien encore : un mouchoir ou un caillou dans la bouche signifiaient que l'homme aurait dû se taire ; les organes génitaux autour du cou qu'il avait harcelé des femmes de mafieux incarcérés. Tandis que la "lettera di scrocco", le pétrole sur les fruits, les animaux étranglés étaient des invitations pressantes à payer le "pizzo". La tête d'un animal près d'une habitation, un cœur en métal troué, un cercueil vide, un oiseau mort étaient quant à eux des avertissements à une mort promise ", in Greco 2011 (nous traduisons). On pourrait également citer le phénomène de la « jambisation » tel qu'il se pratique désormais en France.

14. https://www.cnrtl.fr/definition/silence

15. Idem.

16. Dans la religion, on parle ainsi de Loi ancienne, de Loi nouvelle, des Tables de la Loi.

17. Au sens du Verbum, traduction du Logos par Saint-Jérôme, avec une connotation active, d'action. 
18. Il nous faut bien sûr établir des distinctions à propos de cette loi du silence. Ainsi par exemple, il va de soi que le secret militaire est légitimé par le fait qu'est en jeu la survie d'une Nation. Le devoir de réserve dans certaines professions est quant à lui lié à une déontologie protégeant les individus. Le secret d'État engage les intérêts supérieurs de la Nation. En revanche, dans les sociétés dites secrètes, ou plutôt, dans les faits, discrètes, comme la francmaçonnerie, la loi du silence demeure comme valeur culturelle et rituelle, emprunt d'un fort et ancien traditionalisme symbolique.

19. Sur cette question, nous renvoyons notamment aux ouvrages de Charles Guignebert qui analyse le christianisme comme mystère indicible et notamment le mystère du Christ dans les Epîtres et les Actes des Apôtres, donc dans la tradition paulinienne.

20. Comme on le sait, souvent inspirés de la Bible.

21. Cf. Susini et Champeyrache 2019.

22. Rite initiatique fondé en 1801 , composé de 33 degrés. On distingue les loges des trois premiers degrés des hauts grades (du $4^{\mathrm{e}}$ au $33 \mathrm{e}$ degré).

23. Une foi qui n'implique pas nécessairement de croire mais de suivre. Cette idée rejoint d'ailleurs l'autre étymologie possible du mot omertà qui serait issue de l'arabe el amr wa't'ta'a, signifiant « l'ordre et l'obéissance ».

24. C'est par cette mort que la Parole est perdue. Bien évidemment, les (mes)aventures de la Loge P2 n'ont pas manqué, en Italie notamment, de susciter à ce sujet de nombreux commentaires, plus ou moins autorisés.

25. 1 Cor. $13: 12$.

26. Sur la question de la violence qui suscite ou engendre de la violence et sur la violence qui produit du sacré, nous renvoyons aux ouvrages de René Girard.

27. Dans ce cas, la philia est l'alliance à un groupe, le neikos l'action criminelle. Il ne pourrait y avoir de clan familial, par exemple sans l'idée, même détournée, de philia.

28. Dumoulié 2019, p. 105.

29. N'est-il pas de meilleure communication verbale et non verbale que celle qui peut agir sur le pouvoir de l'imagination? Si la communication mafieuse se joue entre action et silence, entre violence et sacré, c'est un imaginaire où l'esprit va chercher à satisfaire un besoin d'identification et de puissance.

30. Nous rejoignons par là le sociologue allemand Georg Simmel et l'une de ses thèses sur la "fonction sociale» du secret qui «n'est pas de l'ordre de l'avoir ni de l'être mais de l'agir », in Simmel 1998, p. 367. Cette forme de «l'agir", de l'action nous renvoie au Verbum comme forme de l'action justement (cf. note 15).

31. Cerullo 2016.

32. Les femmes jouent dans la Camorra un rôle central. Elles commandent, ordonnent des assassinats, tuent, élèvent leurs enfants avec le lait de la pègre et leur demandent, quand le père est en prison, de garder haut l'honneur de la famille.

33. Parmi les innombrables films, il nous faut citer Le Parrain de Francis Ford Coppola, sans doute l'un des premiers grands succès internationaux sur le thème. Ce film nous intéresse particulièrement car il montre bien, par le choix de représenter le parrain mafieux sous les traits d'un des plus beaux acteurs de l'histoire du cinéma, Marlon Brando, par quoi passe l'esthétique de la violence et la violence dans l'esthétique. L'identification avec la beauté esthétique du criminel - que l'on retrouve dans de nombreux films policiers (et l'on songe aussi à Alain Delon) participe à un désir d'identification.

34. Indicatif à ce propos le boom des «mafia tour» ou des "padrino tour", ces circuits touristiques sur les lieux les plus emblématiques de l'histoire mafieuse mais également sur les lieux ayant servi de décors à des films.

35. C'est notamment ce qui fait dire à Davide Cerullo: «Si je pense à la Napoli qu'avait vue Pasolini, celle de Saviano est comme embouteillée dans une boule de verre : tu la mets à l'envers 
et la neige tombe, souvenir pour touristes. Saviano est tout le contraire de Pasolini : il est limité, manque d'humilité, il est apparence. ", in Cerullo 2016, p. 98 (nous traduisons).

36. Cf. Banet 2012.

37. Cf. notamment le récent débat suscité par les propos sur la Rai de Leonardo Zappalà et Niko Pandetta au sujet de Giovanni Falcone et Paolo Borsellino.

38. «Certains neomelodici ont explicitement glorifié la mafia. Il mio amico camorrista de Lisa Castaldi en est un parfait exemple: "Mon ami le truand» chante t-elle, "risque sa vie et sa liberté / Mais pour les gens de la rue, il n'y a pas d'autre loi ». La chanson 'O capo clan de Nello Liberti est une apologie similaire de la loi mafieuse : «Le boss est un homme sérieux, il ne souhaite pas le mal / S'il a fauté c'était par nécessité et en accord avec la volonté de Dieu ». En 2015, Umberto Accurso un de ces boss qui purge actuellement une peine de dix ans de prison - a écrit une chanson pour son fils, intitulée À la liberté, détaillant la peine qu'on ressent lorsqu'on est séparé de ses êtres chers. Elle a été chantée à la télévision locale par Anthony, un des plus célèbres neomelodici de Naples », in Mackay 2016.

\section{ABSTRACTS}

Cet article vise à s'interroger sur la façon dont les mafias se construisent et s'enracinent dans le tissu socio-culturel en se fondant avec et dans le mythologique, et à montrer comment la mythologie mafieuse ou le halo mythologique des mafias sont profondément inscrits dans leur processus communicationnel, jusqu'à créer un « désir de mafia ».

Questo studio esamina le modalità messe in opera dalle mafie per costruirsi e radicarsi nel tessuto socio-culturale fondendosi col e nel mitologico. Analizza inoltre la mitologia mafiosa e l'aura mitologica elaborate dalle mafie nell'ambito dei loro processi comunicativi, tanto da generare un vero e proprio « desiderio di mafia ».

\section{INDEX}

Mots-clés: mythe, loi du silence, hommes d'honneur, silence et sacré, sémiologie

\section{AUTHOR}

\section{LAURENT LOMBARD}

Laurent Lombard est maître de conférences (habilité à diriger des recherches) en littérature italienne contemporaine à l'Université d'Avignon. Il a traduit en français près de 80 ouvrages, parmi lesquels on peut citer des romans d'Antonio Moresco, Massimo Carlotto et Igort. Récemment, il a développé le concept de «traducteur polytrope » pour pallier les manques et les défauts de la traductologie. 